\title{
Design and Simulation of Ultrasensitive Nano-Biosensor Based on OFPC
}

\author{
Mehdi NEJADEBRAHIMY*, Lida HALIMI, and Hamed ALIPOUR-BANAEI \\ Department of Electronics, College of Engineering, Tabriz Branch, Islamic Azad University, Tabriz, Iran \\ *Corresponding author: Mehdi NEJADEBRAHIMY E-mail: stu.ebrahimy@iaut.ac.ir
}

\begin{abstract}
We designed and simulated a nano-biosensor to work in wet chemical optical processes for the determination and analysis of gaseous or liquid media. For this purpose, the optical properties of materials have been studied, and by creating the relationship between the refractive index of materials and other optical parameters, the measurement process was carried out. In this work, an optical filter based on the photonic crystal (OFPC) was used. By creating an active environment for the interaction between the substance and electromagnetic light, a situation to measure the properties of available substances in that active environment could be provided. Considering that the defect created in the OFPC may cause disruption in its operation, so the volume of the environment should be limited. Creation of defects in the structure of the nano-biosensors can increase the accuracy and quality of measurements; finally by rearranging the created defects, the output will be placed in the appropriate scope. The accuracy is increased by applying the finite difference time domain (FDTD) modeling approach in order to analyze the wave equations governing the structure of the photonics crystal.
\end{abstract}

Keywords: Nano-biosensor, OFPC, $Q$-factor, sensitivity, FDTD

Citation: Mehdi NEJADEBRAHIMY, Lida HALIMI, and Hamed ALIPOUR-BANAEI, "Design and Simulation of Ultrasensitive Nano-Biosensor Based on OFPC," Photonic Sensors, 2015, 5(1): 43-49.

\section{Introduction}

The rapid growth of the science and technology requires human to dominate over the environment. Therefore, it is necessary to make sensors with the high quality and precision. Different methods have been introduced to achieve this purpose [1-16]. One of these technologies is the use of photonic crystal structures [3-5]. Photonic crystals are periodic structures made of dielectric materials that are divided into three structures: 1D, 2D, and 3D based on their refractive index distribution functions [11]. Because 1D structures lack a complete band gap and it is too difficult to make 3D structures due to a small constant lattice [6], therefore, 2D structures are used because they have the complete band gap and easier to make the 2D structure rather than the $3 \mathrm{D}$ one. Usually, there are two modes in $2 \mathrm{D}$ structures: air holes in a dielectric substrate and dielectric cylindrical rods immersed in air. Optical filters based on the photonic crystal are prepared by creating an environment for the formation of the resonance. This can be done by creating the defects in the photonic crystal structure [1]. By changing the filter parameters, structure characteristics such as the quality factor, output gain, and wavelength can be managed. S. H. Kwon et al. proposed a photonic crystal chemical sensor based on a cavity [12].

Received: 12 June 2014 / Revised version: 15 July 2014

(C) The Author(s) 2014. This article is published with open access at Springerlink.com

DOI: $10.1007 / \mathrm{s} 13320-014-0211-2$

Article type: Regular 
There are two types of mechanisms to design biosensors: one method is homogeneous measurement by which changes in the environment refractive index are measured, and the other method is superficial measurement by which the thickness changes in the surface level due to the environmental changes are measured [7]. In optical sensors, the parameter of measurement sensitivity plays an important role in determining the measurement accuracy of the sensor [5]. This feature can be improved by increasing the quality factor of the sensor. The interesting features of photonic crystals for sensor designers are integrative capability, and there is possibility of design sensors in nano scales; so these structures can be used for bio-chemical applications such as measuring the characteristics of molecules, protein tissues, and DNA [17].

The goal of this study was to design a nanobiosensor for measuring the chemical processes. In order to do this, an optical filter based on the photonic crystal (OFPC) structure based on a cavity resonator was designed in the form of air holes in the dielectric substrate. To communicate between substances dispersed in space and electromagnetic waves using from the OFPC, an active environment within the resonance cavity was created. The wavelength chosen by the resonant cavity was a function of the dispersed substance in the active environment. The measurement accuracy increased by improving the active environment of the sensor. Considering that an extensive increase in the volume of this environment makes the output of filter unstable and non-linear, therefore a mediocre portion was considered. Using the critical data, the measurement process became linear; then the accuracy of sensor measurement was studied by the use of the finite difference time domain (FDTD) modeling.

\section{Theoretical modeling and data analysis}

The first step in designing optical filters is the optimal design of the band structure of photonic crystals [5]. This is achieved by determining the photonic band gap (PGB) [1]. The photonic band gap (PBG) is referred to a range of wavelengths according to their distributions of dispersion, and a range of electromagnetic waves don not propagate within their structures. This range is used for designing the OFPC. The design model is a hexagonal photonic crystal structure that contains air holes in the dielectric substrate with the effective refractive index of 2.53 , dimensions of $25 * 25$ cells, constant lattice of $570 \mathrm{~nm}$, and radius of $180 \mathrm{~nm}$. The photonic crystal band structure is illustrated in Fig. 1. For the proposed structure, a PBG is found only for the TM polarization. It is shown that the PBG is in the range of $160 \mathrm{THz}<F<208 \mathrm{THz}$.

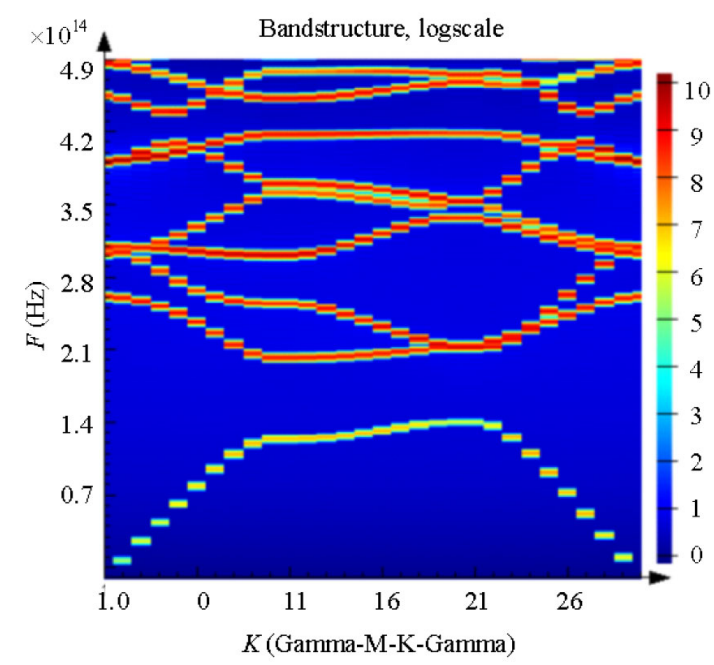

Fig. 1 Band structure of the photonic crystal.

Three following parts are formed by creating basic defects in the structure of the OFPC:

(1) Input waveguide

(2) Output waveguide

(3) Resonance cavity

Input and output waveguides in the OFPC structure transfer the waves from the input to the cavity and from the cavity to the output; this role is loaded on them because the input wavelength is placed in the realm of the PBG. The action of wavelength selection inside the cavity is done in a particular wavelength by creating resonance. The overall outlook of the OFPC after the exertion of 
basic defects is illustrated in Fig. 2. One method for classifying different materials is to divide them according to their refractive indexes.

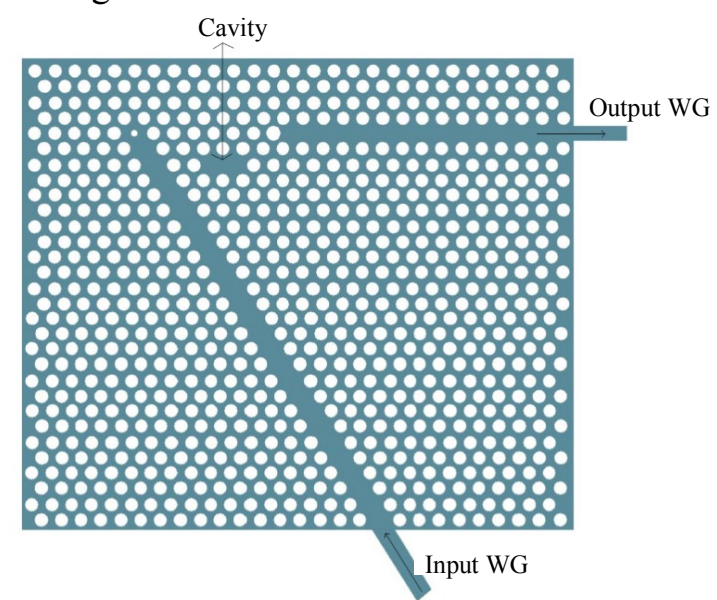

Fig. 2 Overall outlook of the OFPC after the exertion of basic defects.

Thus, if a chemical optical process for designing the biosensor uses the homogeneous measurement mechanism [4], the nature of materials can be recognized according to their optical characteristics.

In the OFPC structure, the wavelength selection is done by specific parameters chosen from the resonance cavity. In other words, by controlling these parameters, scattering inside cavities will change, and this will result in a shift in the resonance wavelength inside the cavity [1]. For instance, some of these parameters are mentioned below:

(1) The geometry of the resonance cavity and topological position in accordance with the input and output waveguides.

(2) A part of cavity: by changing its refractive index, the output wavelength changes.

Thus, if we can create an environment in the active area of the cavity, by preserving the stability of the OFPC, which can affect scattering of electromagnetic waves, the wavelength output of the OFPC and the environments' inner material will be related to each other, and the selected wavelength will be a function of the effective refractive index $n_{\text {analysis }}$ and active area of $\Delta s$ :

$$
\Lambda_{\text {output }} \approx \Delta s^{*} n_{\text {analysis }}
$$

where $n_{\text {analysis }}$ is the complex refractive index, which can be written as $n_{R}+\mathrm{i} k_{I}$, where the real part $n_{R}$ is the conventional index, and the imaginary part $k_{I}$ is the optical extinction coefficient [19]. Considering the changes in the material and the related changes in the refractive index which will result in the changes in the output wavelength, for the accurate operation of the chemical optical process, these changes should be linear. Since the OFPC response is linear just for some limited wavelengths, the chemical optical process should be designed only for that area. Thus, it is just used for measuring materials which have special refractive indexes. The refractive indexes of different materials are shown in Table 1.

Table1 Values of background refractive index.

\begin{tabular}{ccc}
\hline Substance & Wavelength $(\mu \mathrm{m})$ & Refractive index \\
\hline Water & 1.55 & 1.326 \\
Ethanol & 1.55 & 1.3575 \\
Methanol & 1.55 & 1.322 \\
Chloride & 1.55 & 1.425 \\
Butane & 1.55 & 1.3802 \\
Toluene & 1.55 & 1.5 \\
\hline
\end{tabular}

Due to the difference in refractive indexes of the materials listed in Table1, by creating the active area of the OFPC cavity and placing different materials in this area, the effect of changing different materials in the active region on the shift in the output wavelength will be studied. For this aim, the nano-biosensor structure will be optically simulated. One of the methods of photonic structure simulations is the FDTD. This method, which is a scalar method for the Maxwell equations, was first introduced in 1966 by Dr. Yee. In this method, after discretization, the Maxwell equations will be solved in the time and space domains; time steps and space steps should be appropriate. This proportion is known as the covariant stability condition. According to this condition, if a plane wave is distributed in the FDTD network, in each time step, every point of the wave should not pass more than a cell [16]. In the FDTD in each time step, waves just 
can pass from one cell to the next one. For achieving the time step, an orientation will be considered by which the wave in that orientation passes the most cells in the time unit, and this orientation will be perpendicular to the FDTD surface network. For the 2D network dimension, if all dimensions have the same length, width, height of $\Delta u$, and the maximum distribution speed mentioned as $V$, thus the time period $t$ will be obtained from this relationship:

$$
v \Delta t \leqslant \frac{\Delta u}{\sqrt{d}} .
$$

For the 2D structure in the general state, it is

$$
v \Delta t \leqslant \frac{1}{\sqrt{\frac{1}{(\Delta x)^{2}}+\frac{1}{(\Delta z)^{2}}}} .
$$

Generally, the results of the equation are acceptable, and the results that are smaller than $\Delta t$ will not be acceptable. Considering that the measurement process takes place inside the cavity, the active area in the cavity should have a proper stability in sensor's output measurement limit. So the dimensions of the cavity should not be bigger than a particular quantity. The placement and dimensions of the active area of the cavity are shown in Fig. 3. As shown in Fig. 3, the volume of the cavity should be $2 * 10^{-11} \mathrm{~nm}^{2}$, and it should be in the center of the cavity. If we want to put the nano-biosensor output in the proper measurement limit, we should adjust the place of the output peak, measurable refractive index limit, output gain, and quality factor via creating defects in the OFPC structure; for this purpose, $R_{a}, R_{b}$, and $R_{c}$ defects in the OFPC structure will be created. The important parameter that determines the resonance wavelength in the resonant cavity is the radius of introduced defects inside them. The diameters of defects are defined as $R_{c}$ and $R_{b}$. The sensor output wavelength area will be specified in the cavity, so by changing the cells radius $R_{b}$, the output wavelength is controlled, and its placement will change in order of different substances in the active area of the cavity.
Thus, the most proper measurement wavelength should be selected in order to have the best status. The coupled optical powers from the input waveguide to the cavity and from the cavity to the output waveguide are specified by $R_{a}$ and $R_{c}$. By designing the proper radius and their placement, the output power of the sensor quality factor is controlled, and the best condition will be selected. In this sensor, the measurement sensitivity is so important in its efficiency and determines the measurement accuracy.

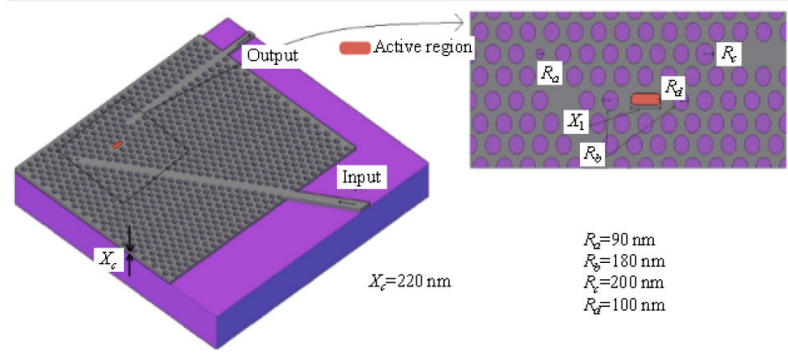

Fig. 3 Placement topology of the active environment in the resonance cavity.

Considering the measurement method in this sensor, by increasing the output wavelength quality factor, the measurement accuracy and stability will be improved, and a little change in the substance type could be identified in the output. The cells radius $R_{c}$ is one of the elements of specifying quality factor in this nano-biosensors which is determined by the situation and measurement limit.

The location and size of defects created in the nano-biosensor structure are shown in Fig. 3. In this part by injecting different substances in the active area of the cavity, the optical simulation is done, and by coupling electromagnetic waves in the input waveguide, the electromagnetic waves with a range of the wavelengths by considering the substance inside the active area of the resonance cavity are coupled to the output waveguide. By changing the substance inside the cavity, the output peak will change, and a specific characteristic of the substance is sensed. The measurement process is shown in Fig. 4. 


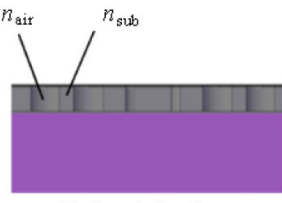

Before injection

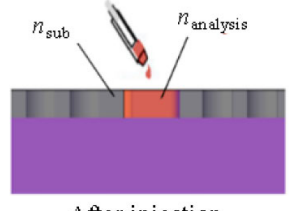

(a)

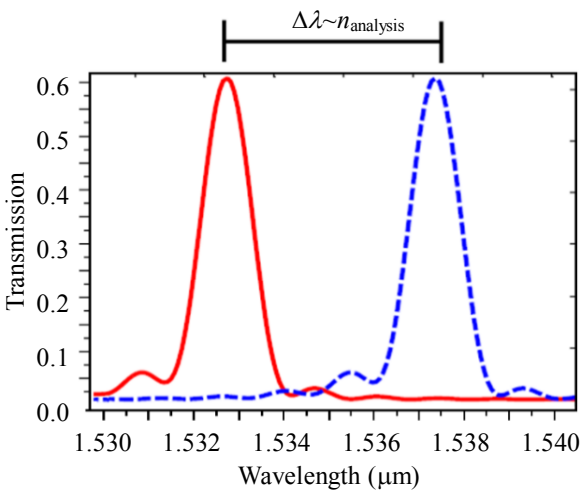

(b)

Fig. 4 Measurement process: (a) substance injection method in the nano-biosensors and (b) blue shift of the optical spectrum induced by pore filling with the substance.

\section{Results and discussion}

In this part, the designed nano-biosensor was tested. For this job, different organic and nano organic substances in liquid or gaseous state were used, and all the changes in the output peak should be noted. Considering the measurement process shown in Fig. 4, the nano-biosensor input range is in a relatively wide range of wavelengths $(90 \mathrm{~nm})$; by considering the output wavelength which passes to the exit, the substance could be specified. If testing substances are modeled in the $n_{R}+\mathrm{i} k_{I}$ material, in other words, both the reflective index and optical losses are taken into account, the quality of measurement will increase. Since the continuity of the output curve of the nano-biosensor is in accordance with different refractive indexes, as shown in Fig. 4(b), in this state, 2 measurable substances combined in the working range of the nano-biosensor are used; considering the ratio of powers of the output peaks of two substances is between the peaks of the measurement states of first and second substances, thus the amount of the shift in the output wavelength will be related to the percentage of components according to the calibrated and established standard states in Fig. 5, also the output figure of the sensor is shown according to various models of the $n_{R}+\mathrm{i} k_{I}$ material [18].

The results for the novel designed nanobiosensor are listed in Table 2. According to this table, $\lambda_{0}$ is the central wavelength, and $\Delta \lambda_{\mathrm{FWHM}}$ is the full width at the half-maximum (FWHM) of the output which indicates the behavior of the narrow band biosensor. According to $\lambda_{0}$ and $\Delta \lambda_{\mathrm{FWHM}}$, the value of quality factors for the nano-biosensor is calculated as $\lambda_{0} / \Delta \lambda_{\mathrm{FWHM}}$, and the transmission efficiency is defined as the division of the output magnetic field amplitude to the input magnetic field amplitude.

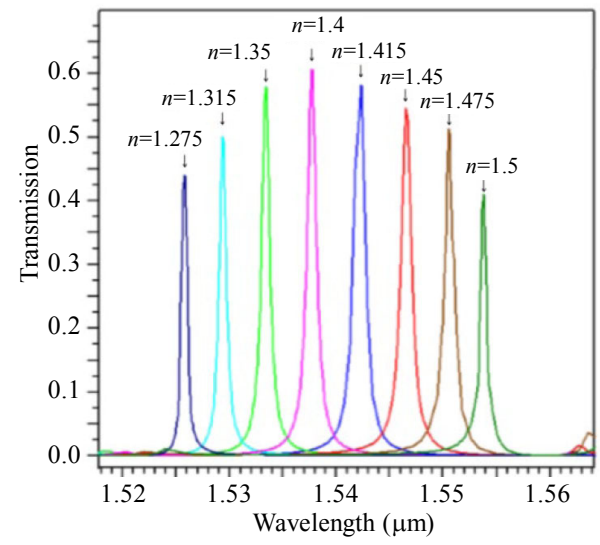

Fig.5 Measurement sensitivity via output wavelength according to normalized refractive indexes.

Table 2 Simulation results of the nano-biosensor with different refractive indexes.

\begin{tabular}{ccccc}
\hline Refractive index & $\lambda_{0}$ & $\Delta \lambda_{\text {FWHM }}$ & Quality factor & Efficiency (\%) \\
\hline 1.275 & 1525 & 0.38 & 4013 & 45 \\
1.315 & 1529 & 0.4 & 3822 & 51 \\
1.35 & 1533 & 0.46 & 3332 & 56 \\
1.4 & 1537.5 & 0.49 & 3137 & 60 \\
1.415 & 1542 & 0.52 & 2965 & 58 \\
1.45 & 1546.5 & 0.54 & 2863 & 54 \\
1.475 & 1551 & 0.55 & 2820 & 51 \\
1.5 & 1554 & 0.5 & 3108 & 40 \\
\hline
\end{tabular}

The optical sensing system was tested with different organic and non-organic solutions used in 
the gaseous or liquid state. As an example, the matching curve of the sensitivity of various organic materials is shown in Fig. 6.

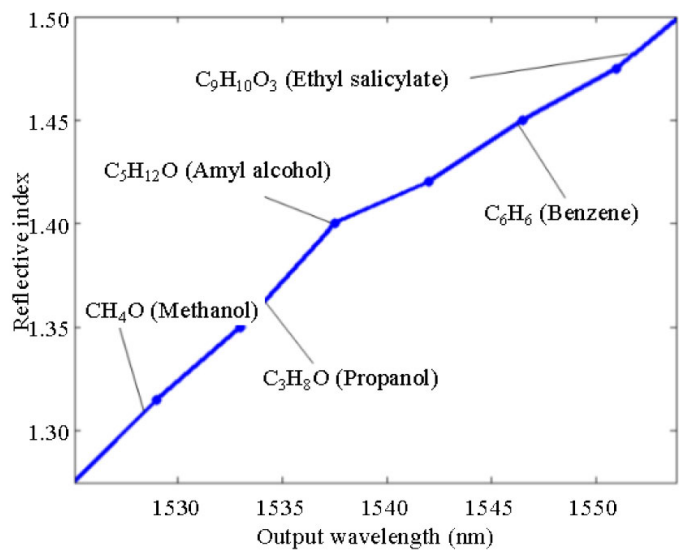

Fig. 6 Matching curve of sensitivity of the nano-biosensor with various organic materials.

\section{Conclusions}

The purpose of our study was to design an optical nano-biosensor for the intrusive measurement in wet chemical optical systems. Since this nano-biosensor's design was based on the OFPC, this structure was designed, and particularly, the parameters were embedded in it for managing the output; so that the OFPC's quality and accuracy could be controlled via these parameters. In this job, the parameters were modified in a way that in the output, the maximum efficiency was achieved by an appropriate quality factor. By creating an active environment in a part of the resonance cavity, an environment for the interaction between substances and electromagnet wave in the measurement process was designed. In continued, different materials in liquid or gaseous state were modeled as $n_{R}+\mathrm{i} k_{I}$ material. The obtained results were analyzed, and the optimum working range for the nano-biosensor was determined.

Open Access This article is distributed under the terms of the Creative Commons Attribution License which permits any use, distribution, and reproduction in any medium, provided the original author(s) and source are credited.

\section{References}

[1] S. Olyaee and A. A. Dehghani, "High resolution and wide dynamic range pressure sensor based on two-dimensional photonic crystal," Photonic Sensors, 2012, 2(1): 92-96.

[2] A. Rostami, H. A. Banaei, F. Nazari, and A. Bahrami, "An ultra-compact photonic crystal wavelength division demultiplexer using resonance cavities in a modified Y-branch structure," Optik - International Journal for Light and Electron Optics, 2011, 122(16): 1481-1485.

[3] F. Hsiao and C. Lee, "Novel biosensor based on photonic crystal nano-ring resonator," Procedia Chemistry, 2009, 1(1): 417-420.

[4] A. Kovacs, A. Malisauskaite, A.Ivanov, U. Mescheder, and R. Wittig, "Optical sensing and analysis system based on porous layers," in 17th International Conference on Miniaturized Systems for Chemistry and Life Sciences, Germany, October 27-31, 2013.

[5] S. Olyaee and M. Azizi, "Micro displacement sensor based on high sensitivity photonic crystal," Photonic Sensors, 2014, 4(3): 220-224.

[6] O. Levy, B. Z. Steinberg, M. Nathan, and A. Boag, "Ultrasensitive displacement sensing using photonic crystal waveguides," Applied Physics Letters, 2005 , 86(10): 104102-104104.

[7] K. Manoharan, "Design and analysis of high-Q, amorphousmicroring resonator sensors for gaseous and biological species detection," M.S. thesis, Russ College of Engineering and Technology of Ohio University, America, 2009.

[8] A. M. Vengsarkar, P. J. Lemaire, J. B. Judkins, V. Bhatia, T. Erdogan, and J. E. Sipe, "Long period gratings as band-rejection filters," Journal of Lightwave Technology, 1996, 14(1): 58-64.

[9] K. Kalli, T. Allsop, K. Zhou, G. Smith, M. Komodromos, D. Webb, et al., "Sensing properties of femtosecond laser-inscribed long period gratings in photonic crystal fiber," Photonic Sensors, 2011, 1(3): 228-233.

[10] M. Qiu, "Effective index method for hetero structureslab-waveguide-based-two-dimensional photonic crystals," Applied Physics Letters, 2002, 81(7): 1163-1165.

[11] J. Sun and C. C. Chan, "Photonic bandgap fiber for refractive index measurement," Sensors and Actuators B: Chemical, 2007, 128(1): 46-50.

[12] S. H. Kwon, T. Sünner, M. Kamp, and A. Forchel, "Optimization of photonic crystal cavity for chemical sensing," Optics Express, 2008, 16(16): 11709-11717.

[13] E. Hallynck and P. Biensman, "Photonic crystal biosensor based on angular spectrum analysis," Optics Express, 2010, 18(17): 18164-18170.

[14] D. Yang, H. Tian, and Y. Ji, "Micro-displacement 
sensor based on high-Q nanocavity in slot photonic crystal," Optical Engineering, 2011, 50(5): 544021-544026.

[15] J. D. Joannopoulos, S. G. Johnson, J. N. Winn, and R. D. Meade, Photonic crystals molding the flow of light. Princeton: Princeton University Press, 2007.

[16] V. Bhatia, "Applications of long-period gratings to single and multi-parameter sensing," Optics Express, 1999, 4(11): 457-466.

[17] V. Toccafondo, J. García-Rupérez, M. J. Bañuls, A. Griol, J. García-Castelló, S.Peransi-Llopis, et al.,
"DNA detection using a photonic crystal waveguide sensor," in Advanced Photonics and Renewable Energy, OSA Technical Digest, 2010.

[18] K. Moutzouris, M. Papamichael, S. C. Betsis, I. Stavrakas, G. Hloupis, and D. Triantis, "Refractive, dispersive and thermo-optic properties of twelve organic solvents in the visible and near infrared," Applied Physics B, 2014, 116(3): 617-622.

[19] J. S. Foresi, P. R. Villeneuve, J. Ferrera, E. R. Thoen, G. Steinmeyer, S. Fan, et al., "Photonicbandgapmicrocavities in optical waveguides," Nature, 1997, 390: 143-145. 